International Journal of Engineering \& Technology, $5(x)(2017) x x x-x x x$
International Journal of Engineering \& Technology
SPC
Website: $w$ ww.sciencepubco.com/index.php/IJET
doi: $x x$

\title{
A New Hyperchaotic Hyperjerk System with Three Nonlinear Terms, its Synchronization and Circuit Simulation
}

\author{
Sundarapandian Vaidyanathan ${ }^{1}$, *Aceng Sambas ${ }^{2}$, Mohamad Afendee Mohamed $^{3}$, Mustafa Mamat ${ }^{3}$ and W. S. \\ Mada Sanjaya ${ }^{4}$ \\ ${ }^{1}$ Research and Development Centre, Vel Tech University, Avadi, Chennai, India \\ ${ }^{2}$ Department of Mechanical Engineering, Universitas Muhammadiyah Tasikmalaya, Indonesia \\ ${ }^{3}$ Faculty of Informatics and Computing, Universiti Sultan Zainal Abidin, Malaysia \\ ${ }^{4}$ Department of Physics, Universitas Islam Negeri Sunan Gunung Djati, Bandung, Indonesia \\ *acengs@umtas.ac.id
}

\begin{abstract}
In recent decades, hyperjerk systems have been studied well in the literature because of their simple dynamics structure and complex qualitative properties. In this work, we announce a new hyperchaotic hyperjerk system with three nonlinear terms. Dynamical properties of the hyperjerk system are analyzed through equilibrium analysis, dissipativity, phase portraits and Lyapunov chaos exponents. We show that the new hyperchaotic hyperjerk system has a unique saddle-focus equilibrium at the origin. Thus, the new hyperchaotic hyperjerk system has a self-excited strange attractor. Next, global hyperchaos synchronization of a pair of new hyperchaotic hyperjerk systems is successfully achieved via adaptive backstepping control. Also, an electronic circuit of the hyperchaotic hyperjerk system has been designed via MultiSIM to check the feasibility of the theoretical system.
\end{abstract}

Keywords: Hyperchaos, hyperchaotic systems, hyperjerk systems, circuit simulation, synchronization.

\section{Introduction}

It is well-known that chaos theory has application has many branches of science and engineering ([1]-[2]). Some popular applications can be mentioned as plasma systems [3], weather systems ([4]-[5]), chemical reactions ([6]-[8]), encryption ([9]-[11]), robotics [12], oscillations ([13]-[16]), circuits ([17]-[20]), etc.

In 1996, it was shown by Gottlieb [21] that 3-D chaotic systems can be expressed in the form of single ordinary differential equations, which are also termed as jerk differential equations. The jerk differential equations arise in many physical models of science and engineering such as jerk circuits [22], thermal arc plasma [23], biological reactions [24], mechanical oscillations [25], etc.

In physics, a jerk differential equation can be represented as the third order dynamics

$$
\dddot{x}=f(x, \dot{x}, \ddot{x}),
$$

where $x(t)$ represents the displacement, $\dot{x}(t)$ the velocity, $\ddot{x}(t)$ the acceleration and $\dddot{x}(t)$ the jerk.

A famous example of a jerk system is the Coullet system [26], which is described by the jerk dynamics

$\dddot{x}+a \ddot{x}+\dot{x}-b\left(x^{2}-1\right)=0$.

In [26], it was established that the jerk dynamics (2) is chaotic when $(a, b)=(0.6,0.58)$
Generalizing the jerk system (2), we obtain the hyperjerk system given by

$D^{(n)}(x)=f\left(x, D x, \ldots, D^{(n-1)}(x)\right),(n \geq 4)$,

where $D=\frac{d}{d t}$ is the time-derivative.

In the modelling of dynamical systems, it is a common practice to express the $n$th order ODE (3) as an equivalent system of $n$ ordinary differential equations.

This is carried out by defining $n$ phase variables defined as follows:

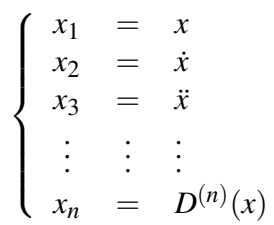

Using the phase variables (4), we can give a representation in system form for the hyperjerk differential equation (3) as follows:

$\left\{\begin{array}{ccc}\dot{x}_{1} & = & x_{2} \\ \dot{x}_{2} & = & x_{3} \\ \vdots & \vdots & \vdots \\ \dot{x}_{n-1} & = & x_{n} \\ \dot{x}_{n} & = & f\left(x_{1}, x_{2}, \ldots, x_{n}\right)\end{array}\right.$ 
Thus, the $n$-th order ODE (3) and the system of $n$ ODEs (5) are equivalent. Henceforth, we shall consider hyperjerk systems in the system form given by Eq. (5). Hyperjerk systems have generated good interest in the literature due to their simple structure and complex qualitative properties ([27]-[31]).

By adding a quadratic nonlinearity to Daltzis hyperjerk system [32] and considering different parameter values, we derive a new hyperchaotic hyperjerk system in this work. In Section 2, we describe the dynamics and qualitative properties of the new hyperchaotic hyperjerk system. As an engineering application, we derive global hyperchaos synchronization results for the new hyperchaotic hyperjerk system with unknown parameters using adaptive backstepping control in Section 3. Furthermore, we design an electronic circuit using MultiSIM for the new hyperchaotic hyperjerk system in Section 4. Conclusions are summarized in Section 5.

\section{A new hyperjerk system with three nonlinear terms}

In 2018, Daltzis et al. [32] presented a new 4-D hyperjerk system given by

$$
\left\{\begin{array}{l}
\dot{x}_{1}=x_{2} \\
\dot{x}_{2}=x_{3} \\
\dot{x}_{3}=x_{4} \\
\dot{x}_{4}=-x_{1}-x_{2}-a x_{3}-b\left|x_{2}\right|-c x_{1}^{4} x_{4}
\end{array}\right.
$$

where $a, b$ and $c$ are positive parameters. In [32], it was shown that the Daltzis hyperjerk system (6) is hyperchaotic when the parameters take the values $(a, b, c)=(3.8,0.1,1.5)$.

Using Wolf's algorithm [33], the Lyapunov exponents of the Daltzis hyperjerk system (6) are obtained for $(a, b, c)=(3.8,0.1,1.5)$ and $X(0)=(0.1,0.1,0.1,0.1)$ for $T=1 E 4$ seconds as

$L_{1}=0.1201, L_{2}=0.0210, L_{3}=0, L_{4}=-1.2854$.

Since $L_{1}$ and $L_{2}$ are positive in (7), we conclude that the Daltzis hyperjerk system (6) is hyperchaotic.

Also, the Kaplan-Yorke dimension of the Daltzis hyperjerk system (6) is calculated as

$D_{K Y}=3+\frac{L_{1}+L_{2}+L_{3}}{\left|L_{4}\right|}=3.1098$,

which gives a pointer to the complexity of the Daltzis hyperjerk system (6).

In this paper, we propose a new 4-D hyperjerk system by adding a quadratic nonlinearity to Daltzis hyperjerk system (6) as follows.

$$
\left\{\begin{array}{l}
\dot{x}_{1}=x_{2} \\
\dot{x}_{2}=x_{3} \\
\dot{x}_{3}=x_{4} \\
\dot{x}_{4}=-x_{1}-x_{2}-a x_{3}-b\left|x_{2}\right|-c x_{1}^{4} x_{4}-d x_{2}^{2}
\end{array}\right.
$$

where $a, b, c$ and $d$ are positive parameters.

We show that the system (9) is hyperchaotic for the parameter values $(a, b, c, d)=(3.8,0.01,1.3,0.05)$.

Using Wolf's algorithm [33], the Lyapunov exponents of the new hyperjerk system (9) are obtained for $(a, b, c, d)=(3.8,0.01,1.3,0.05)$ and $X(0)=(0.1,0.1,0.1,0.1)$ for $T=1 E 4$ seconds as

$L_{1}=0.1251, L_{2}=0.0183, L_{3}=0, L_{4}=-1.1275$

Since $L_{1}$ and $L_{2}$ are positive Lyapunov exponents, the new hyperjerk system (9) is hyperchaotic.

By adding all Lyapunov exponents in (10), we get the sum as -0.9841 , which is negative. This shows that the new hyperjerk system (9) is dissipative.
The Kaplan-Yorke dimension of the new hyperjerk system (9) is determined as

$D_{K Y}=3+\left(L_{1}+L 2+L_{3}\right) /\left|L_{4}\right|=3.1272$,

which gives a pointer to the high complexity of the hyperchaotic hyperjerk system (9).

The maximal Lyapunov exponent (MLE) of the new hyperjerk system (9) is $L_{1}=0.1251$, which is greater than the MLE of the Daltzis hyperjerk system (6) given by $L_{1}=0.1201$.

Also, the Kaplan-Yorke dimension of the new hyperjerk system (9) is $D_{K Y}=3.1272$, which is greater than the Kaplan-Yorke dimension of the Daltzis hyperjerk system (6) given by $D_{K Y}=3.1098$. Thus, we have shown that the new hyperjerk system (9) is more complex than the Daltzis hyperjerk system (6).

The equilibrium points of the new hyperjerk system (9) are tracked by solving the following equations:

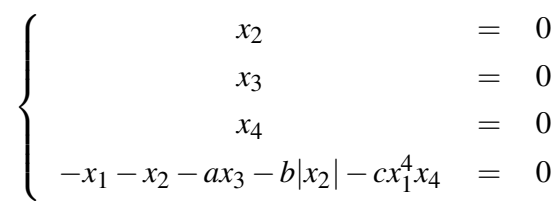

For all the parameter values, the new hyperjerk system (9) has a unique equilibrium point at the origin:

$E_{0}=\mathbf{0}=\left[\begin{array}{l}0 \\ 0 \\ 0 \\ 0\end{array}\right]$

The Jacobian matrix of the new hyperjerk system (9) at the equilibrium point $E_{0}=\mathbf{0}$ is obtained as

$J_{0}=\left[\begin{array}{cccc}0 & 1 & 0 & 0 \\ 0 & 0 & 1 & 0 \\ 0 & 0 & 0 & 1 \\ 0 & 0 & -a & 0\end{array}\right]$

For the hyperchaotic case, the parameters are taken as $(a, b, c, d)=$ $(3.8,0.01,1.3,0.05)$.

Then we have

$J_{0}=\left[\begin{array}{cccc}0 & 1 & 0 & 0 \\ 0 & 0 & 1 & 0 \\ 0 & 0 & 0 & 1 \\ 0 & 0 & -3.8 & 0\end{array}\right]$

which has the eigenvalues $0,0, \pm 1.9494 i$.

Thus, the new hyperjerk system (9) has a critical case at the origin. The stability of the new hyperjerk system (9) can be further investigated using Lyapunov stability theory.

The phase portraits of the new hyperchaotic hyperjerk system (9) are displayed in Figures 1-4.

The Lyapunov exponents of the new hyperchaotic hyperjerk system (9) are shown in Figure 5.

\section{Global hyperchaos synchronization of the new hyperjerk systems}

In this section, we study an engineering application of the new hyperjerk system, viz. global hyperchaos synchronization of a pair of new hyperjerk systems considered as master and slave systems via adaptive backstepping control method. 


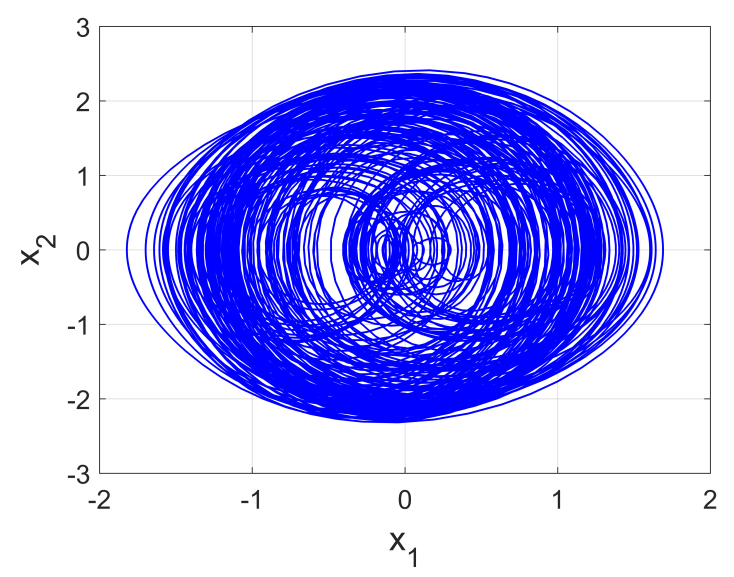

Figure 1: MATLAB simulations of phase portraits of the new hyperchaotic hyperjerk system (9) for $X(0)=(0.1,0.1,0.1,0.1)$ and $(a, b, c, d)=$ $(3.8,0.01,1.3,0.05)$ in $\left(x_{1}, x_{2}\right)$ plane

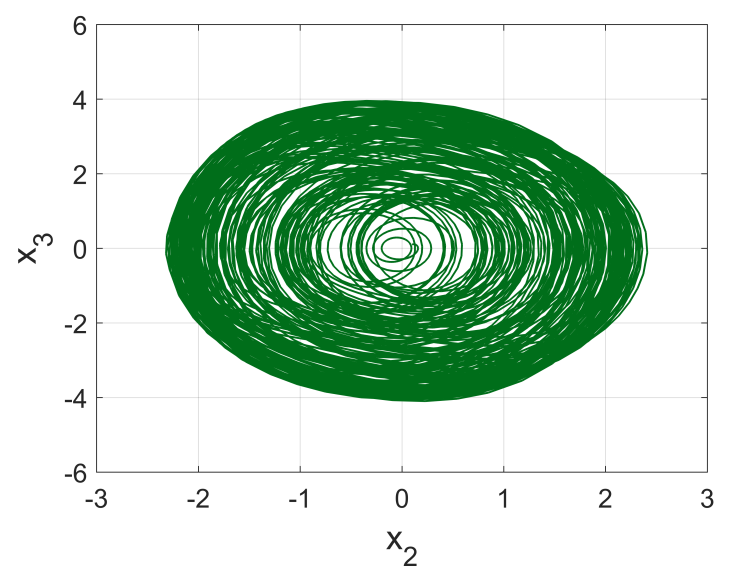

Figure 2: MATLAB simulations of phase portraits of the new hyperchaotic hyperjerk system (9) for $X(0)=(0.1,0.1,0.1,0.1)$ and $(a, b, c, d)=$ $(3.8,0.01,1.3,0.05)$ in $\left(x_{2}, x_{3}\right)$ plane

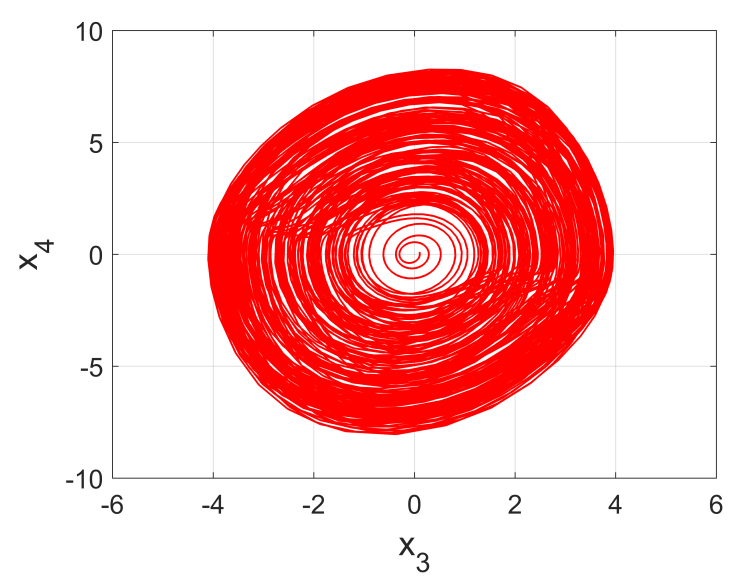

Figure 3: MATLAB simulations of phase portraits of the new hyperchaotic hyperjerk system (9) for $X(0)=(0.1,0.1,0.1,0.1)$ and $(a, b, c, d)=$ $(3.8,0.01,1.3,0.05)$ in $\left(x_{3}, x_{4}\right)$ plane

As the master system, we consider the new hyperjerk system given

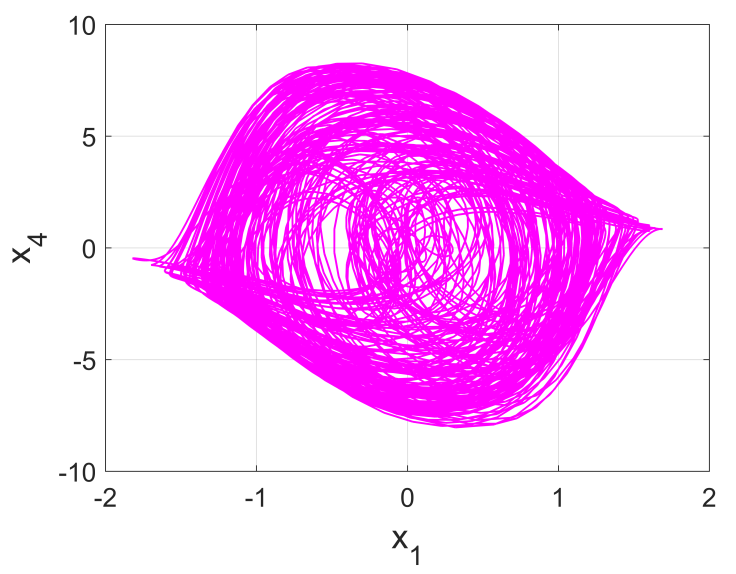

Figure 4: MATLAB simulations of phase portraits of the new hyperchaotic hyperjerk system (9) for $X(0)=(0.1,0.1,0.1,0.1)$ and $(a, b, c, d)=$ $(3.8,0.01,1.3,0.05)$ in $\left(x_{1}, x_{4}\right)$ plane

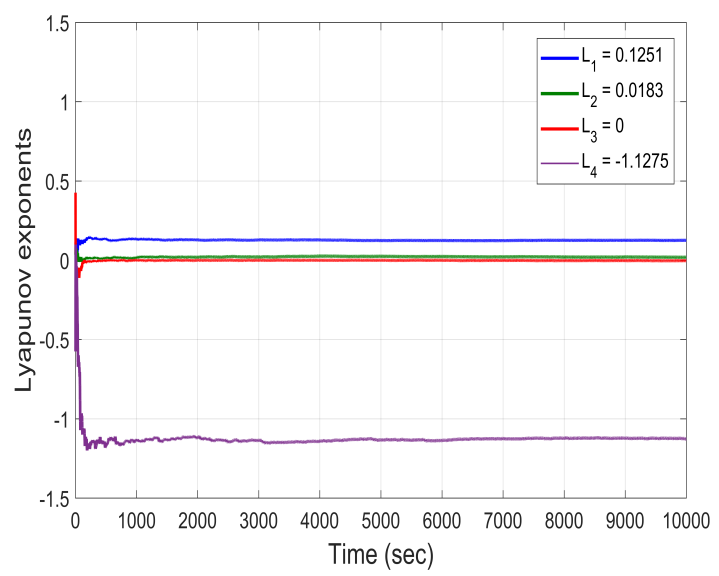

Figure 5: Lyapunov exponents of the new hyperchaotic hyperjerk system (9) for $X(0)=(0.1,0.1,0.1,0.1)$ and $(a, b, c, d)=(3.8,0.01,1.3,0.05)$

by

$$
\left\{\begin{array}{l}
\dot{x}_{1}=x_{2} \\
\dot{x}_{2}=x_{3} \\
\dot{x}_{3}=x_{4} \\
\dot{x}_{4}=-x_{1}-x_{2}-a x_{3}-b\left|x_{2}\right|-c x_{1}^{4} x_{4}-d x_{2}^{2}
\end{array}\right.
$$

where $x_{1}, x_{2}, x_{3}, x_{4}$ are the states and $a, b, c, d$ are unknown parameters of the system.

As the slave system, we consider the new hyperjerk system given by

$$
\left\{\begin{array}{l}
\dot{y}_{1}=y_{2} \\
\dot{y}_{2}=y_{3} \\
\dot{y}_{3}=y_{4} \\
\dot{y}_{4}=-y_{1}-y_{2}-a y_{3}-b\left|y_{2}\right|-c y_{1}^{4} y_{4}-d y_{2}^{2}+u
\end{array}\right.
$$

where $y_{1}, y_{2}, y_{3}, y_{4}$ are the states and $u$ is the backstepping control to be found.

The synchronization error between the new hyperjerk systems (16) and (17) is defined by

$\left\{\begin{array}{l}e_{1}=y_{1}-x_{1} \\ e_{2}=y_{2}-x_{2} \\ e_{3}=y_{3}-x_{3} \\ e_{4}=y_{4}-x_{4}\end{array}\right.$ 
The error dynamics is calculated as follows.

$$
\left\{\begin{aligned}
\dot{e}_{1}= & e_{2} \\
\dot{e}_{2}= & e_{3} \\
\dot{e}_{3}= & e_{4} \\
\dot{e}_{4}= & -e_{1}-e_{2}-a e_{3}-b\left(\left|y_{2}\right|-\left|x_{2}\right|\right) \\
& -c\left(y_{1}^{4} y_{4}-x_{1}^{4} x_{4}\right)-d\left(y_{2}^{2}-x_{2}^{2}\right)+u
\end{aligned}\right.
$$

Next, we define the estimation errors for the unknown parameters as

$$
\left\{\begin{array}{l}
e_{a}(t)=a-A(t) \\
e_{b}(t)=b-B(t) \\
e_{c}(t)=c-C(t) \\
e_{d}(t)=d-D(t)
\end{array}\right.
$$

where $A(t), B(t), C(t), D(t)$ are estimates for $a, b, c, d$, respectively. Differentiating (20), we get

$$
\left\{\begin{array}{l}
\dot{e}_{a}(t)=-\dot{A}(t) \\
\dot{e}_{b}(t)=-\dot{B}(t) \\
\dot{e}_{c}(t)=-\dot{C}(t) \\
\dot{e}_{d}(t)=-\dot{D}(t)
\end{array}\right.
$$

Using adaptive backstepping control method, we establish the key result of this section.

Theorem 1. The master and slave hyperchaotic systems represented by the new hyperjerk systems (16) and (17) with unknown parameters are globally and exponentially synchronized by means of the adaptive backstepping feedback control law given by

$$
\left\{\begin{aligned}
u= & -4 e_{1}-9 e_{2}-(9-A(t)) e_{3}-4 e_{4}+B(t)\left(\left|y_{2}\right|-\left|x_{2}\right|\right) \\
& +C(t)\left(y_{1}^{4} y_{4}-x_{1}^{4} x_{4}\right)+D(t)\left(y_{2}^{2}-x_{2}^{2}\right)-k \xi_{4}
\end{aligned}\right.
$$

where $k>0$ is a gain constant,

$\xi_{4}=3 e_{1}+5 e_{2}+3 e_{3}+e_{4}$

and the update law for the parameter estimates $A(t), B(t), C(t), D(t)$ is given by

$$
\left\{\begin{array}{l}
\dot{A}(t)=-\xi_{4} e_{3} \\
\dot{B}(t)=-\xi_{4}\left(\left|y_{2}\right|-\left|x_{2}\right|\right) \\
\dot{C}(t)=-\xi_{4}\left(y_{1}^{4} y_{4}-x_{1}^{4} x_{4}\right) \\
\dot{D}(t)=-\xi_{4}\left(y_{2}^{2}-x_{2}^{2}\right)
\end{array}\right.
$$

Proof. We establish this result via adaptive backstepping contorl method and Lyapunov stability theory [34].

We define the Lyapunov function

$V_{1}\left(\xi_{1}\right)=\frac{1}{2} \xi_{1}^{2}$

where

$\xi_{1}=e_{1}$

Differentiating $V_{1}$ along the error dynamics (19), we get

$\dot{V}_{1}=\xi_{1} \dot{\xi}_{1}=e_{1} e_{2}=-\xi_{1}^{2}+\xi_{1}\left(e_{1}+e_{2}\right)$

We set

$\xi_{2}=e_{1}+e_{2}$

Using (28), we can simplify Eq. (27) as

$\dot{V}_{1}=-\xi_{1}^{2}+\xi_{1} \xi_{2}$
Next, we define the Lyapunov function

$V_{2}\left(\xi_{1}, \xi_{2}\right)=V_{1}\left(\xi_{1}\right)+\frac{1}{2} \xi_{2}^{2}=\frac{1}{2}\left(\xi_{1}^{2}+\xi_{2}^{2}\right)$

Differentiating $V_{2}$ along the error dynamics (19), we get

$\dot{V}_{2}=-\xi_{1}^{2}-\xi_{2}^{2}+\xi_{2}\left(2 e_{1}+2 e_{2}+e_{3}\right)$

We set

$\xi_{3}=2 e_{1}+2 e_{2}+e_{3}$

Using (32), we can simplify Eq. (31) as

$\dot{V}_{2}=-\xi_{1}^{2}-\xi_{2}^{2}+\xi_{2} \xi_{3}$

Next, we define the Lyapunov function

$V_{3}\left(\xi_{1}, \xi_{2}, \xi_{3}\right)=V_{2}\left(\xi_{1}, \xi_{2}\right)+\frac{1}{2} \xi_{3}^{2}=\frac{1}{2}\left(\xi_{1}^{2}+\xi_{2}^{2}+\xi_{3}^{2}\right)$

Differentiating $V_{3}$ along the error dynamics (19), we get

$\dot{V}_{3}=-\xi_{1}^{2}-\xi_{2}^{2}-\xi_{3}^{2}+\xi_{3}\left(3 e_{1}+5 e_{2}+3 e_{3}+e_{4}\right)$

We set

$\xi_{4}=3 e_{1}+5 e_{2}+3 e_{3}+e_{4}$

Using (36), we can simplify Eq. (35) as

$\dot{V}_{3}=-\xi_{1}^{2}-\xi_{2}^{2}-\xi_{3}^{2}+\xi_{3} \xi_{4}$

To simplify the notation, we set $\xi=\left(\xi_{1}, \xi_{2}, \xi_{3}, \xi_{4}\right)$.

Finally, we define the quadratic Lyapunov function

$\left\{\begin{aligned} V\left(\xi, e_{a}, e_{b}, e_{c}, e_{d}\right)= & V_{3}\left(\xi_{1}, \xi_{2}, \xi_{3}\right)+\frac{1}{2} \xi_{4}^{2} \\ & +\frac{1}{2}\left(e_{a}^{2}+e_{b}^{2}+e_{c}^{2}+e_{d}^{2}\right)\end{aligned}\right.$

Clearly, $V$ is a quadratic and positive definite function on $\mathbf{R}^{8}$.

Differentiating $V$ along the error dynamics (19) and (24), we get

$\dot{V}=-\sum_{i=1}^{4} \xi_{i}^{2}+\xi_{4}\left(\xi_{4}+\xi_{3}+\dot{\xi}_{4}\right)-e_{a} \dot{A}-e_{b} \dot{B}-e_{c} \dot{C}-e_{d} \dot{D}$

Eq. (39) can be written compactly as

$\dot{V}=-\sum_{i=1}^{4} \xi_{i}^{2}+\xi_{4} S-e_{a} \dot{A}-e_{b} \dot{B}-e_{c} \dot{C}-e_{d} \dot{D}$

where

$S=\xi_{4}+\xi_{3}+\dot{\xi}_{4}=\xi_{4}+\xi_{3}+\left(3 \dot{e}_{1}+5 \dot{e}_{2}+3 \dot{e}_{3}+\dot{e}_{4}\right)$

A simple computation gives the result

$\left\{\begin{aligned} S= & 4 e_{1}+9 e_{2}+(9-a) e_{3}+4 e_{4}-b\left(\left|y_{2}\right|-\left|x_{2}\right|\right) \\ & -c\left(y_{1}^{4} y_{4}-x_{1}^{4} x_{4}\right)-d\left(y_{2}^{2}-x_{2}^{2}\right)+u\end{aligned}\right.$

Substituting the value of $u$ from (22) into Eq. (42), we get

$\left\{\begin{aligned} S= & -[a-A(t)] e_{3}-[b-B(t)]\left(\left|y_{2}\right|-\left|x_{2}\right|\right) \\ & -[c-C(t)]\left(y_{1}^{4} y_{4}-x_{1}^{4} x_{4}\right) \\ & -[d-D(t)]\left(y_{2}^{2}-x_{2}^{2}\right)-k \xi_{4}\end{aligned}\right.$

Using the definition of parameter estimation errors given in Eq. (20), we can simplify Eq. (43) as follows:

$S=-e_{a} e_{3}-e_{b}\left(\left|y_{2}\right|-\left|x_{2}\right|\right)-e_{c}\left(y_{1}^{4} y_{4}-x_{1}^{4} x_{4}\right)-e_{d}\left(y_{2}^{2}-x_{2}^{2}\right)-k \xi_{4}$ 
Substituting the value of $S$ from Eq. (44) into Eq. (40), we get

$$
\left\{\begin{aligned}
\dot{V}= & -\xi_{1}^{2}-\xi_{2}^{2}-\xi_{3}^{2}-(1+k) \xi_{4}^{2}+e_{a}\left[-\xi_{4} e_{3}-\dot{A}\right] \\
& +e_{b}\left[-\xi_{4}\left(\left|y_{2}\right|-\left|x_{2}\right|\right)-\dot{B}\right] \\
& +e_{c}\left[-\xi_{4}\left(y_{1}^{4} y_{4}-x_{1}^{4} x_{4}\right)-\dot{C}\right] \\
& +e_{d}\left[-\xi_{4}\left(y_{2}^{2}-x_{2}^{2}\right)-\dot{D}\right]
\end{aligned}\right.
$$

Substituting the parameter update law from Eq. (24) into Eq. (45), we get

$$
\dot{V}=-\xi_{1}^{2}-\xi_{2}^{2}-\xi_{3}^{2}-(1+k) \xi_{4}^{2}
$$

which is a negative semi-definite function on $\mathbf{R}^{8}$.

Thus, by Barbalat's lemma in Lyapunov stability theory [34], we conclude that $\mathbf{e}(t)$ is globally exponentially stable. Hence, it is consequent that the master and slave hyperchaotic systems represented by the new hyperjerk systems (16) and (17) are globally and exponentially synchronized for all initial conditions $\mathbf{x}(0), \mathbf{y}(0) \in \mathbf{R}^{4}$. Hence, the proof is complete.

For numerical simulations, we take the parameter values of the new hyperjerk systems (16) and (17) as in the hyperchaotic case, i.e. $(a, b, c, d)=(3.8,0.01,1.3,0.05)$. We take the positive gain constant $k$ as $k=10$.

We take the initial state of the master hyperjerk system (16) as $X(0)=(0.9,-0.7,1.4,0.3)$ and the initial state of the slave hyperjerk system (17) as $Y(0)=(-1.5,0.1,2.7,-1.2)$. The initial conditions of the parameter estimates are taken as $A(0)=6.5, B(0)=4.3$, $C(0)=9.7$ and $D(0)=8.2$.

Figures 6-9 show the complete synchronization of the hyperjerk systems (16) and (17). Figure 10 shows the time-history of the hyperchaos synchronization error $\mathbf{e}=\left(e_{1}, e_{2}, e_{3}, e_{4}\right)$.

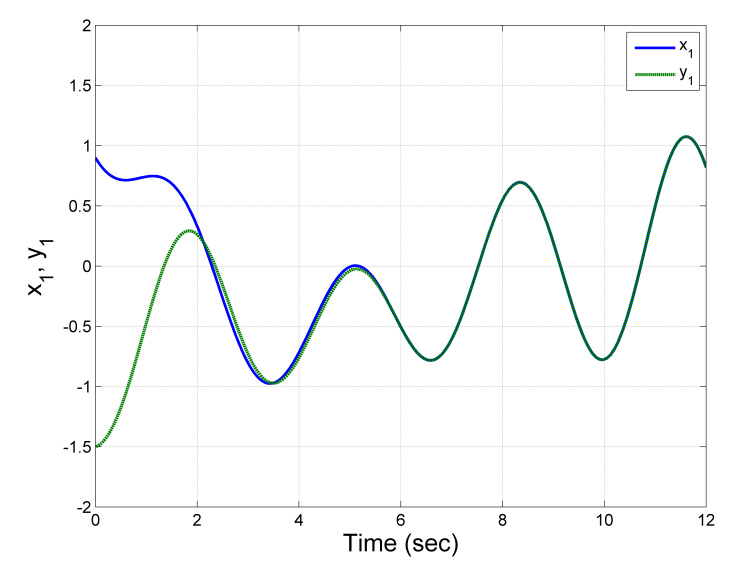

Figure 6: Synchronization of the states $x_{1}$ and $y_{1}$ for the hyperjerk systems (16) and (17)

\section{Circuit design for the new hyperjerk system}

In this section,we design and build an electronic circuit of the new hyperjerk system (9) as shown in Fig. 11. The circuit in Fig. 11 is designed by using operational amplifiers where the state variables $x_{1}, x_{2}, x_{3}$, and $x_{4}$ of new hyperjerk system (9) are associated with the voltages across the capacitors $C_{1}, C_{2}, C_{3}$, and $C_{4}$, respectively. It consists of simple electronic elements, such as resistors, capacitors, operational amplifiers, diode, and analog devices AD633 multipliers. By applying Kirchhoff's laws to this circuit, its dynamics are

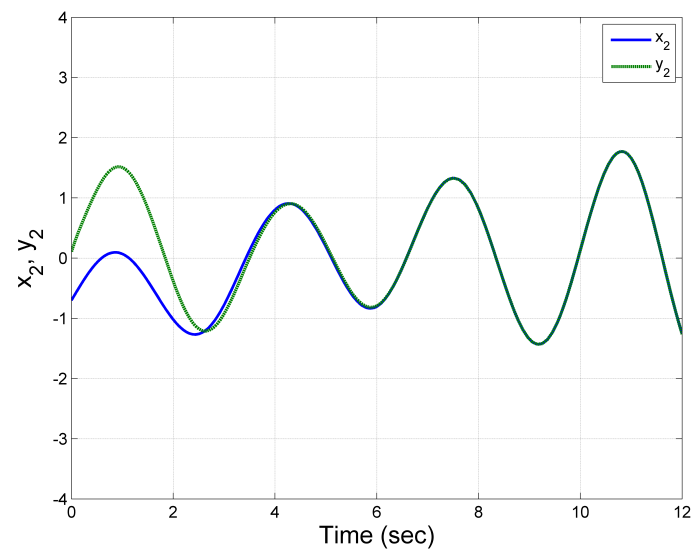

Figure 7: Synchronization of the states $x_{2}$ and $y_{2}$ for the hyperjerk systems (16) and (17)

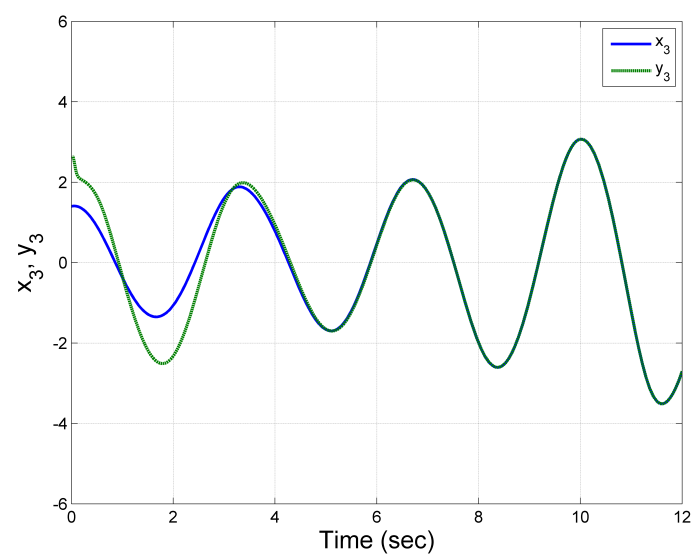

Figure 8: Synchronization of the states $x_{3}$ and $y_{3}$ for the hyperjerk systems (16) and (17)

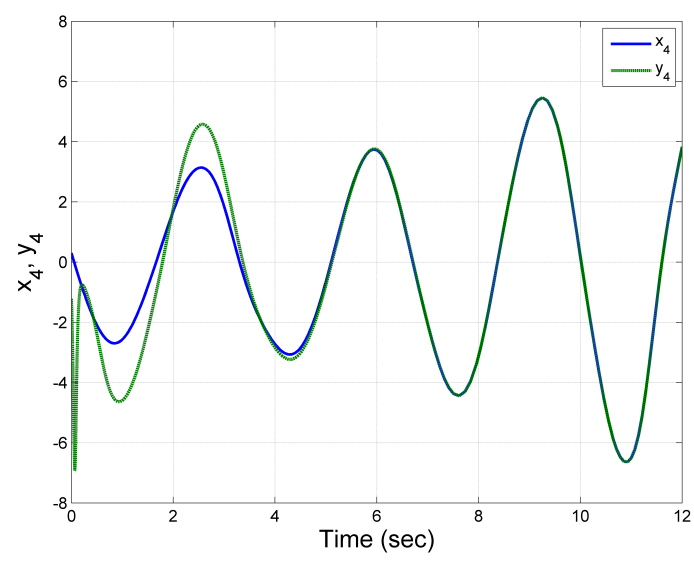

Figure 9: Synchronization of the states $x_{4}$ and $y_{4}$ for the hyperjerk systems (16) and (17)

described by the following circuital equations:

$$
\begin{aligned}
\frac{d V c_{1}}{d t}= & \frac{1}{C_{1} R_{1}} V c_{2} \\
\frac{d V c_{2}}{d t}= & \frac{1}{C_{2} R_{2}} V c_{3} \\
\frac{d V c_{3}}{d t}= & \frac{1}{C_{3} R_{3}} V c_{4} \\
\frac{d V c_{4}}{d t}= & -\frac{1}{C_{4} R_{4}} V c_{1}-\frac{1}{C_{4} R_{5}} V c_{2}-\frac{1}{C_{4} R_{6}} V c_{3}-\frac{1}{C_{4} R_{7}}\left|V c_{2}\right| \\
& -\frac{1}{100 C_{4} R_{8}} V c_{1}^{4} V c_{4}-\frac{1}{C_{4} R_{9}} V c_{2}^{2}
\end{aligned}
$$




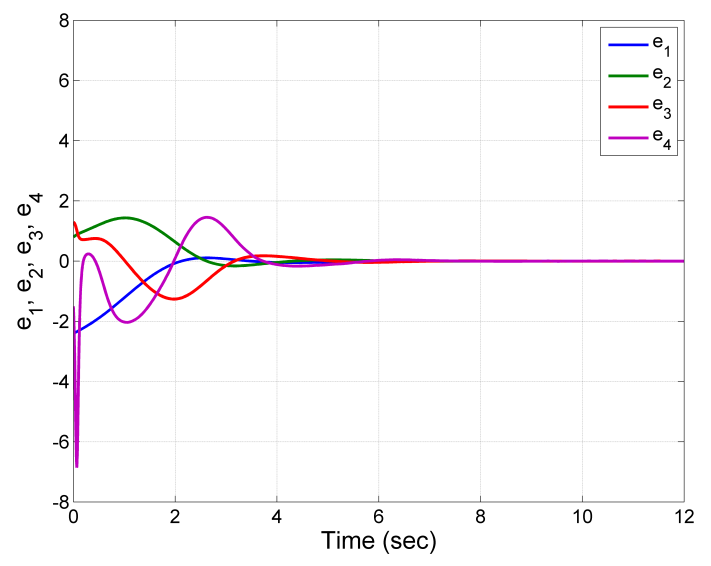

Figure 10: Time-history of the synchronization error e between the hyperjerk systems (16) and (17)

where $V c_{1}, V c_{2}, V c_{3}$, and $V c_{4}$ are the voltages across the capacitors $C_{1}, C_{2}, C_{3}$, and $C_{4}$ respectively. The values of electronic components $R_{6}=26.315 \mathrm{k} \Omega, R_{7}=10 \mathrm{M} \Omega, R_{8}=769 \Omega, R_{9}=2 \mathrm{M} \Omega, R_{1}=$ $R_{2}=R_{3}=R_{4}=R_{5}=R_{10}=R_{11}=R_{12}=R_{13}=R_{14}=R_{15}=R_{16}=$ $R_{17}=R_{18}=R_{19}=R_{20}=100 \mathrm{k} \Omega$, and $C_{1}=C_{2}=C_{3}=C_{4}=1$ $\mathrm{nF}$. The power supplies of all active devices are $\pm 15 \mathrm{~V}$ and the operational amplifiers TL082CD are used. Phase portrait outputs of the electronic circuit simulation are shown in Fig. 12. It can be concluded that good qualitative agreement with the MATLAB simulations is obtained, as well.

\section{Conclusion}

In this paper, based on mathematical models and numerical simulations we have described the dynamics and chaos control of a new hyperjerk system (9) with one absolute nonlinearity. Its chaotic features are fully examined by eigen value structure, Lyapunov exponents and Lyapunov dimension. In addition, an adaptive backstepping controller is introduced to stabilize such hyperjerk system and achieve global hyperchaos synchronization. The designed circuit has been implemented and tested using the MultiSIM software to verify the simulation results. We have found a good agreement between numerical simulations and analog circuit.

\section{Acknowledgement}

The authors thank the Government of Malaysia for funding this research under the Fundamental Research Grant Scheme (FRGS/1/2017/ICT03/Unisza/02/2-RR229) and also Universiti Sul$\tan$ Zainal Abidin, Terengganu, Malaysia.

\section{References}

[1] S. Vaidyanathan and C. Volos, Advances and Applications in Chaotic Systems, Springer, Berlin, (2017).

[2] A.T. Azar and S. Vaidyanathan, Advances in Chaos Theory and Intelligent Control, Springer, Berlin, (2017).

[3] S. Vaidyanathan, "Synchronization of Tokamak systems with symmetric and magnetically confined plasma via adaptive control", International Journal of ChemTech Research, Vol. 8, No. 6, (2015), pp. 818-827.

[4] S. Rasappan and S. Vaidyanathan, "Global chaos synchronization of WINDMI and Coullet chaotic systems by backstepping control", Far East Journal of Mathematical Sciences, Vol. 67, No. 2, (2012), pp. 265-287.

[5] S. Vaidyanathan, C.K. Volos, K. Rajagopal, I.M. Kypriaindis and I.N. Stouboulos, "Adaptive backstepping controller design for the antisynchronization of identical WINDMI chaotic systems with unknown parameters and its SPICE implementation", Journal of Engineering and Technology Review, Vol. 8, No. 2, (2015), pp. 74-82.
[6] S. Vaidyanathan, "A novel chemical chaotic reactor system and its adaptive control", International Journal of ChemTech Research, Vol. 8 No. 7, (2015), pp. 146-158.

[7] S. Vaidyanathan, "Adaptive synchronization of novel 3-D chemical chaotic reactor systems", International Journal of ChemTech Research, Vol. 8, No. 7, (2015), pp. 159-171.

[8] S. Vaidyanathan, "Global chaos synchronization of chemical chaotic reactors via novel sliding mode control method", International Journal of ChemTech Research, Vol. 8, No. 7, (2015), pp. 209-221.

[9] Y.R. Bai, D. Baleanu and G.C. Wu, "A novel shuffling technique based on fractional chaotic maps", Optik, Vol. 168, (2018), pp. 553-562.

[10] G.C. Wu, D. Baleanu and Z.X. Lin, "Image encryption technique based on fractional chaotic time series", Journal of Vibration and Control, Vol. 22, No. 8 (2014), pp. 2092-2099.

[11] S. Vaidyanathan, A. Sambas, M. Mamat and M. Sanjaya WS, "Analysis, synchronisation and circuit implementation of a novel jerk chaotic system and its application for voice encryption", International Journal of Modelling, Identification and Control, Vol. 28, No. 2, (2017), pp. $153-166$.

[12] S. Vaidyanathan, A. Sambas, M. Mamat and M. Sanjaya WS, "A new three-dimensional chaotic system with a hidden attractor, circuit design and application in wireless mobile robot", Archives of Control Sciences Vol. 27, No. 4, (2017), pp. 541-554.

[13] S. Vaidyanathan, "Adaptive controller and synchronization design for the Qi-Chen chaotic system", Lecture Notes of the Institute for Computer Sciences, Social-Informatics and Telecommunications Engineering, Vol. 85, (2012), pp. 124-133.

[14] S. Vaidyanathan, C.K. Volos and V.T. Pham, "Global chaos control of a novel nine-term chaotic system via sliding mode control", Studies in Computational Intelligence, Vol. 576, (2015), pp. 571-590.

[15] S. Vaidyanathan and S. Rasappan, "Hybrid synchronization of hyperchaotic Qi and Lü systems by nonlinear control", Communications in Computer and Information Science, Vol. 131, (2011), pp. 585-593.

[16] V.T. Pham, C.K. Volos and S. Vaidyanathan, "Multi-scroll chaotic oscillator based on a first-order delay differential equation", Studies in Computational Intelligence, Vol. 581, (2015), pp. 59-72.

[17] A. Sambas, S. Vaidyanathan, M. Mamat and W.S. Mada Sanjaya, "A six-term novel chaotic system with hidden attractor and its circuit design", Studies in Systems, Decision and Control, Vol. 133, (2018), pp. 365-373.

[18] S. Vaidyanathan, V.T. Pham, C. Volos and A. Sambas, "A novel 4-D hyperchaotic rikitake dynamo system with hidden attractor, its properties, synchronization and circuit design", Studies in Systems, Decision and Control, Vol. 133, (2018), pp. 345-364.

[19] B.A. Idowu, S. Vaidyanathan, A. Sambas, O.I. Olusola and O.S. Onma, "A new chaotic finance system: Its analysis, control, synchronization and circuit design", Studies in Systems, Decision and Control, Vol. 133, (2018), pp. 271-295.

[20] C.K. Volos, V.T. Pham, S. Vaidyanathan, I.M. Kyprianidis and I.N. Stouboulos, "Synchronization phenomena in coupled Colpitts circuits", Journal of Engineering Science and Technology Review, Vol. 8, No. 2, (2015), pp. 142-151.

[21] H.P.W. Gottlieb, "What is the simplest jerk function that gives chaos?", American Journal of Physics, Vol. 64, (1996), pp. 525.

[22] R. Tchitnga, T. Nguazon, P.H. Louodop Fotso and J.A.C. Gallas, "Chaos in a single op-amp-based jerk circuit: Experiments and simulations", IEEE Transactions on Circuits and Systems II: Express Briefs, Vol. 63, No. 3, (2016), pp. 239-243.

[23] S. Ghorui, S.N. Sahasrabudhe, P.S.S. Murthy, A.K. Das and N. Venkatramani, "Experimental evidence of chaotic behavior in atmospheric pressure arc discharge", IEEE Transactions on Plasma Science, Vol. 28, No. 1, (2000), pp. 253-260.

[24] S. Vaidyanathan, "Super-twisting sliding mode control of the enzymessubstrates biological chaotic system", Studies in Computational Intelligence, Vol. 709, (2017), pp. 435-450.

[25] S. Vaidyanathan, "A seven-term novel jerk chaotic system and its adaptive control", Studies in Computational Intelligence, Vol. 636, (2016), pp. 137-161.

[26] P. Coullet, C. Tresser and A. Arneodo, "Transition to stochasticity for a class of forced oscillators", Physics Letters A, Vol. 72, (1979), pp 268-270.

[27] P. Daltzis, S. Vaidyanathan, V.T. Pham, C. Volos, E. Nistazakis and G Tombras, "Hyperchaotic attractor in a novel hyperjerk system with two nonlinearities", Circuits, Systems, and Signal Processing, Vol. 37, No. 2, (2018), pp. 613-635.

[28] K.E. Chlouverakis and J.C. Sprott, "Chaotic hyperjerk systems", Chaos Solitons and Fractals, Vol. 28, (2006), pp. 739-746.

[29] S. Vaidyanathan, C. Volos, V.T. Pham and K. Madhavan, "Analysis, adaptive control and synchronization of a novel 4-D hyperchaotic hyperjerk system and its SPICE implementation", Archives of Control Sciences, Vol. 25, No. 1, (2015), pp. 135-158.

[30] X. Wang, S. Vaidyanathan, C. Volos, V.T. Pham and T. Kapitaniak, "Dynamics, circuit realization, control and synchronization of a hyperchaotic hyperjerk system with coexisting attractors", Nonlinear Dynamics, Vol. 89, No. 3, (2017), pp. 1673-1687.

[31] V.T. Pham, S. Vaidyanathan, C. Volos, X. Wang and D.V. Hoang, "A hyperjerk memristive system with hidden attractors", Studies in Computational Intelligence, Vol. 701, (2017), pp. 59-80. 
[32] P.A. Daltzis, C.K. Volos, H.E. Nistazakis, A.D. Tsigopoulos and G.S. Tombras, "Analysis, synchronization and circuit design of a 4D hyperchaotic hyperjerk system", Computation, Vol. 6, No. 1, (2018), Article

[33] A. Wolf, J. B. Swift, H. L. Swinney and J. A. Vastano, "Determining Lyapunov exponents from a time series", Physica D: Nonlinear Phenomena, Vol. 16, No. 3, (1985), pp. 285-317.

[34] H.K. Khalil, Nonlinear Systems, Prentice Hall of India, New Jersey, USA (2002). 


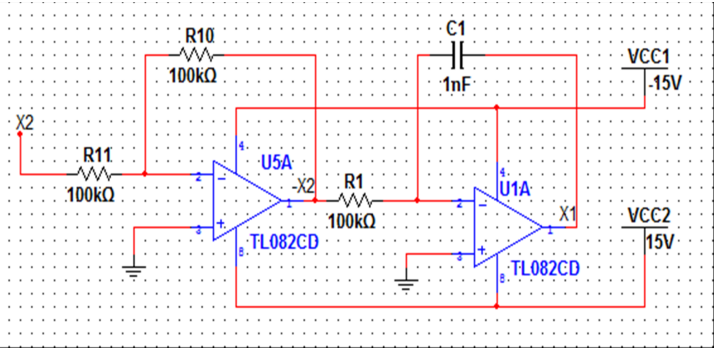

(a)

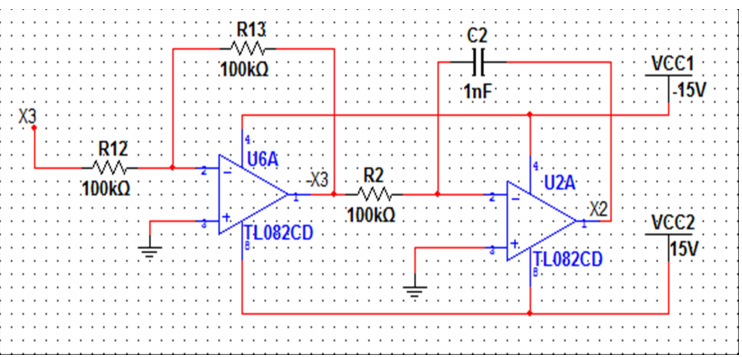

(b)

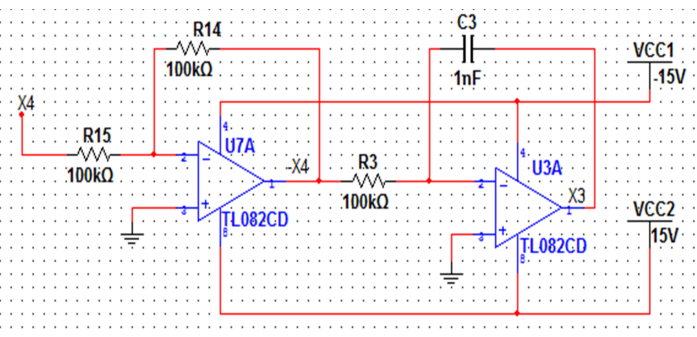

(c)

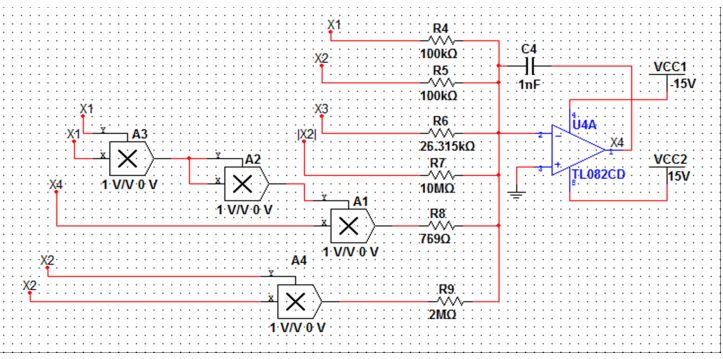

(d)

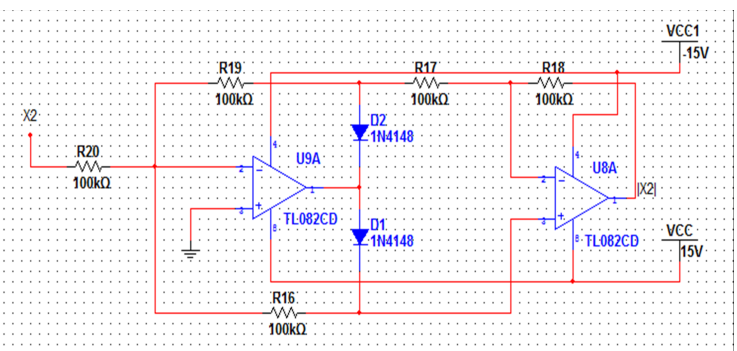

(e)

Figure 11: Circuit design of the new hyperchaotic hyperjerk system (9) (a) $X_{1}$ signal,(b) $X_{2}$ signal, (c) $X_{3}$ signal, (d) $X_{4}$ signal and (e) $\left|X_{2}\right|$ signal

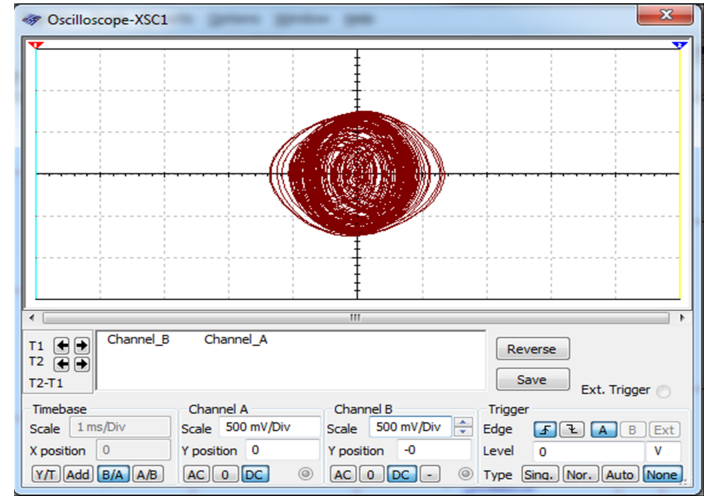

(a)

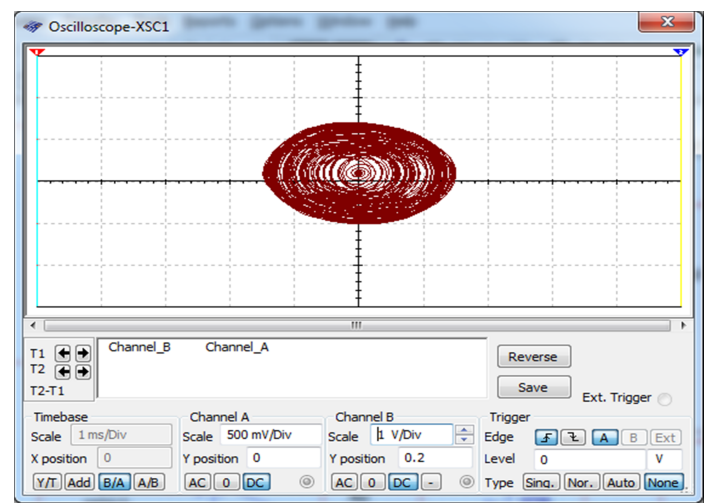

(b)

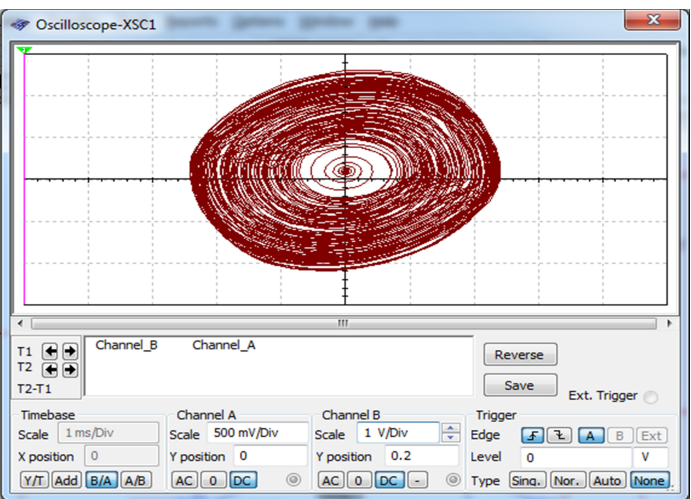

(c)

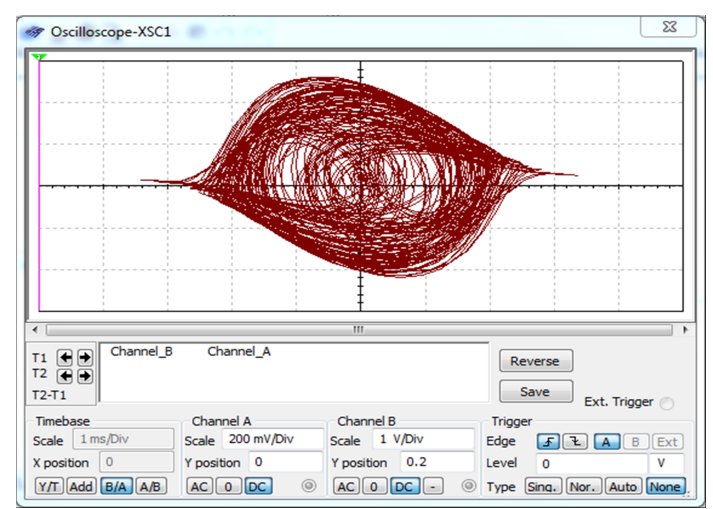

(d)

Figure 12: Chaotic attractors of the new hyperchaotic hyperjerk system (9) using Multisim circuit simulation: (a) $x_{1}-x_{2}$ plane, (b) $x_{2}-x_{3}$ plane, (c) $x_{3}-x_{4}$ plane and (d) $x_{1}-x_{4}$ plane 